\section{Band-selective C- or L-band erbium-doped fiber amplified spontaneous emission source}

\author{
Xiulin Wang \\ Jimei University \\ Department of Physics \\ Xiamen, 361021, China \\ Wencai Huang \\ Xiamen University \\ Department of Electronic Engineering \\ Xiamen, 361005, China
}

\begin{abstract}
Two novel methods are demonstrated to build up a band-selective amplified spontaneous emission (ASE) broadband light source that can operate in the selective conventional band (C-band, $1525 \sim 1565 \mathrm{~nm}$ ) or longwavelength band (L-band, $1565 \sim 1605 \mathrm{~nm}$ ) region using a $1 \times 2$ optical switch. With the backward ASE feedback technique, an output power of $27.6 \mathrm{~mW}$ for the C-band ASE and $11.8 \mathrm{~mW}$ for the L-band ASE are obtained while with the compound pump technique, a power of $24.5 \mathrm{~mW}$ for L-band ASE and $27.2 \mathrm{~mW}$ for C-band ASE are obtained both with a fixed total pump power of $100 \mathrm{~mW}$. The advantage of using the backward ASE feedback technique is that the ASE source design is simpler whereas the power of L-band ASE is lower, and the notable advantage of using the compound pump technique is that the output powers of the two selective bands ( $\mathrm{C}$ - or L-band) are balanced though the configuration is a little complicated. (๑) 2006 Society of Photo-Optical Instrumentation Engineers.

[DOI: 10.1117/1.2171952]
\end{abstract}

Subject terms: fluorescence; erbium lasers; optical communications; fiber optics.

Paper 050651LR received Aug. 18, 2005; revised manuscript received Dec. 6, 2005; accepted for publication Dec. 9, 2005; published online Feb. 13, 2006.

\section{Introduction}

Amplified spontaneous emission (ASE) incoherent broadband light sources based on erbium-doped fiber (EDF) have been a topic of continuing research because of their wide applications for dense wavelength division multiplexing (DWDM) device characterization, spectrum sliced DWDM systems, fiber-optic gyroscopes, and laser spectroscopy. ${ }^{1-3}$ In the past decades, various designs of ASE sources based on EDF have been proposed and investigated with the aim of improving performance parameters such as operating bandwidth, mean-wavelength stability, power density, and conversion efficiency to meet the application requirements. ${ }^{4-8}$

In this paper, we propose and demonstrate an ASE source design that can operate in the selective C- or L-band region with the use of an optical switch. The selective ASE source provides users the necessary band (C- or L-band) when needed without any form of upgrading or additional

0091-3286/2006/\$22.00 @ 2006 SPIE module attachment. Therefore, the selective dual-band ASE fiber source is desirable for various applications in some areas like characteristic measurement for the dual-band DWDM components, and has potential use as a selective upstream or downstream spectrum-sliced coarse WDM transceiver in the emerging metro networks.

\section{Operational Principle and Experimental Results}

\subsection{Band Selection Using the Backward ASE Feedback Technique}

Figure 1 shows the proposed selective dual-band ASE source configuration using the backward ASE feedback technique. The ASE source is composed of a 1480-nm laser diode (LD), two sections of EDF separated by an isolator (ISOI), a 50:50 fiber coupler, a $1 \times 2$ optical switch, a broadband fiber mirror (BBR) simply made by a $3-\mathrm{dB}$ fiber coupler, and an optical isolator (ISOII) at the output port used to avoid lasing of oscillation.

The principle of the band selection can be described as follows. When the optical switch is switched to port 1, the output of the design is a combination of the single-pass backward ASE and the single-pass forward ASE. Since the single-pass forward L-band ASE is weak, the output spectrum is mainly of the backward ASE in C-band region. When the optical switch is switched to port 2, the backward ASE is reflected back to the gain region. Namely, the design becomes a double-pass forward configuration which provides an L-band ASE spectrum. Therefore, by switching the optical switch from port 1 to port 2, the operational band of the ASE design can be selected between C-band and L-band. Namely, the C-band ASE is obtained without backward ASE feedback while the L-band ASE is obtained with the backward ASE feedback. Here, the 1480-nm LD is selected as the pump laser by considering that the 1480-nm LD is more helpful to obtain higher power L-band ASE than that by $980-n m$ LD. It should be noted that the ISOI is necessary in this design. The isolator in between the two fiber sections ensures that the backward L-band ASE from the long fiber (EDFII) does not shift the ASE spectrum of the short fiber section (EDFI). Therefore, the C-band ASE will be obtained when the optical switch switched to port 1. It has been shown that the output spectrum of backward ASE is always C-band with a 980-nm LD as the pump laser no matter how long the EDF is used. ${ }^{6}$ Therefore, the ASE design can be simplified to one-stage configuration by se-

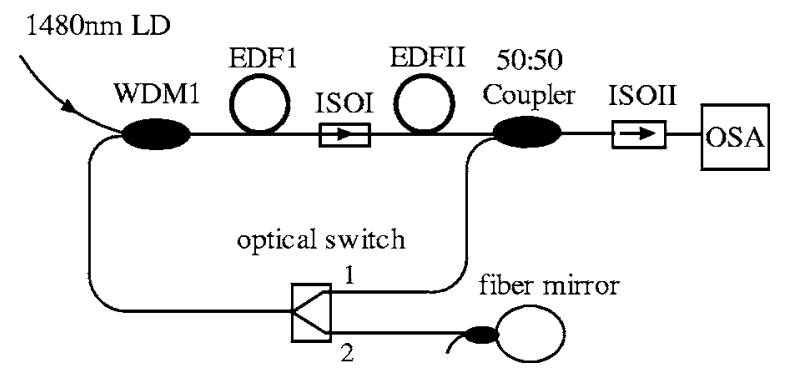

Fig. 1 Schematic diagram of the band-selective ASE source using the backward ASE feedback technique. 
lecting a 980-nm LD as the pump laser but the output power of L-band ASE will be much lower than that by 1480-nm pump LD.

In our experiments, the EDF lengths of first and second stages are 5 and $14 \mathrm{~m}$, respectively, of heavily doped erbium fiber with a peak absorption of $22-33 \mathrm{~dB} / \mathrm{m}$ at $1530 \mathrm{~nm}$. Such lengths are chosen due to the following reason: the 5-m length of section one ensures that it is highly inverted when the optical switch is switched to port 1, so a C-band ASE will be obtained, and the 19-m length of both sections (namely the 14-m length of section two) ensures that the output ASE spectrum shifts to the L-band when the optical switch is switched to port 2 .

Figure 2 shows the output C-band ASE spectrum when the optical switch is switched to port 1 . The resolution of OSA is set at $0.1 \mathrm{~nm}$ for all the spectral measurements. It can be seen from Fig. 2 that the output spectrum falls on the C-band. Its output power is measured to be $27.6 \mathrm{~mW}$ with a total pump power of $100 \mathrm{~mW}$. When the optical switch is switched to port 2, while keeping the parameters of the design unchanged, the output ASE spectrum switches to L-band, as shown in Fig. 3. The output power of the L-band ASE is measured to be $11.8 \mathrm{~mW}$.

\subsection{Band Selection Using the Compound Pump Technique}

It has been shown above that the band selective ASE source could be obtained by controlling the operation state of the optical switch in Fig. 1. Namely, the C-band ASE is obtained without backward ASE feedback while the L-band ASE is obtained with the backward ASE feedback when the optical switch operates at two different states. The ASE configuration and its operational principle are very simple. However, similar to that of Ref. 9, the power of the L-band ASE is much lower compared to that of the C-band ASE since the double-pass forward (DPF) configuration responds to generating the L-band ASE. Apparently, a bandselective ASE source with balanced output power of the C-band and the L-band could be more useful in applications. Previous research has pointed out that the doublepass bidirectional pumped configuration is the best one for

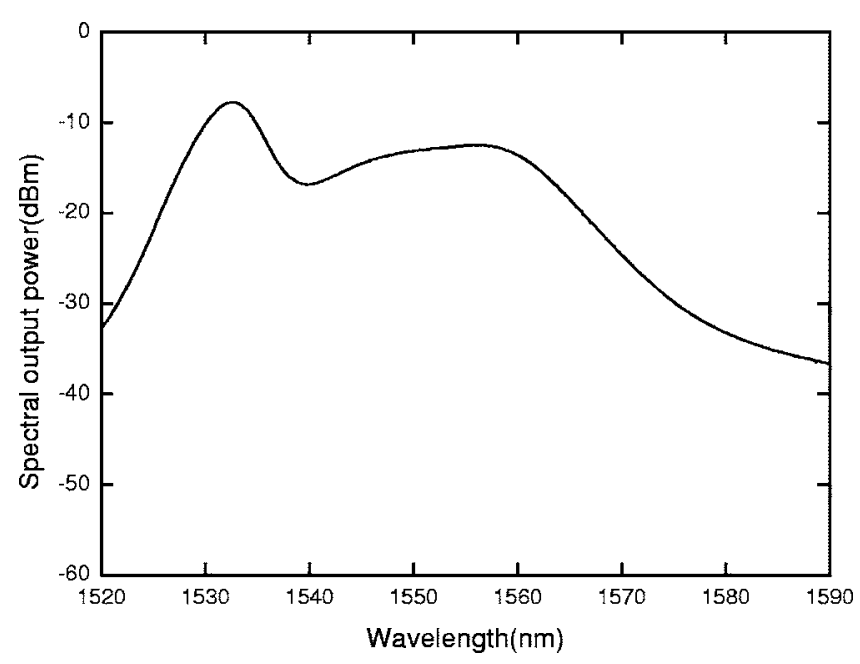

Fig. 2 Output C-band ASE spectrum when the optical switch is switching to port 1.

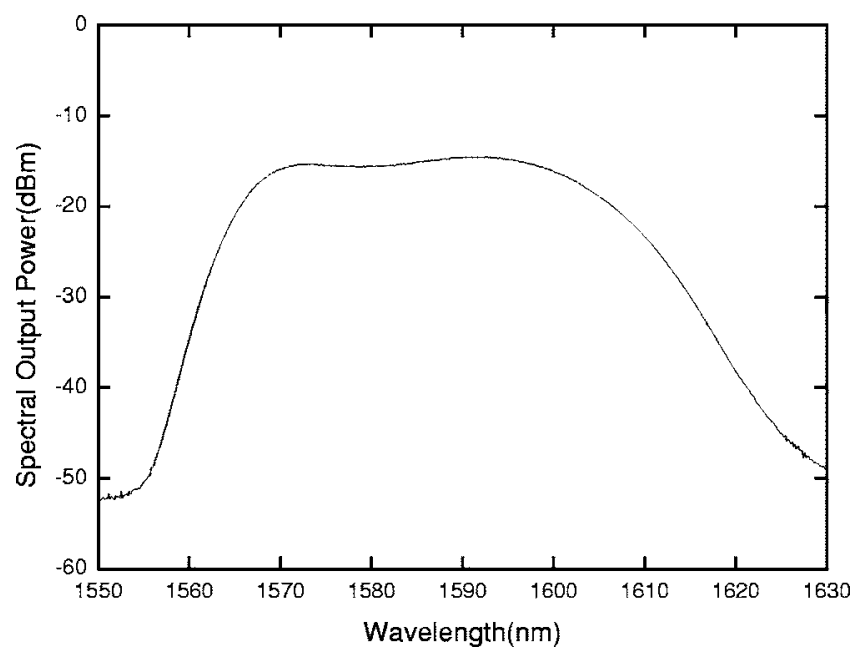

Fig. 3 Output L-band ASE spectrum when the optical switch is switching to port 2.

generating the L-band ASE with a pumping-conversion ef-

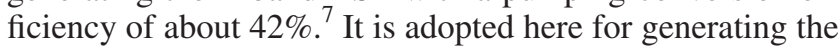
L-band ASE to enhance the power of L-band ASE. The backward pumped configuration is also used for generating the C-band ASE. Therefore, the selective C or L-band ASE source operates in the $\mathrm{C}$-band without forward pumping whereas in the L-band it operates with forward pumping with the use of an optical switch.

Figure 4 is the schematic diagram of the proposed ASE source design that used the compound pump technique. It is composed of two sections of EDF separated by an isolator (ISOI), a $1 \times 2$ optical switch, a power combiner, a broadband fiber mirror (BBR), and an optical isolator (ISOII) at the output port used to avoid lasing of oscillation. The source is also pumped with a single 100-mW, 1480-nm pump laser diode. The pump output is split into two portions using a 50:50 fused fiber splitter. Two-selective-band operation of the C- or L-band ASE output is determined by the operation state of the $1 \times 2$ optical switch. When the optical switch is switched to port 1 , the design becomes a bidirectional pumped configuration with 50:50 pump power from forward and backward synchronously. This configuration ensures the output ASE spectrum falls on L-band as follows: the weak L-band ASE generated by the forward pump is amplified by C-band ASE generated by the backward pump; this causes energy loss and wavelength shift

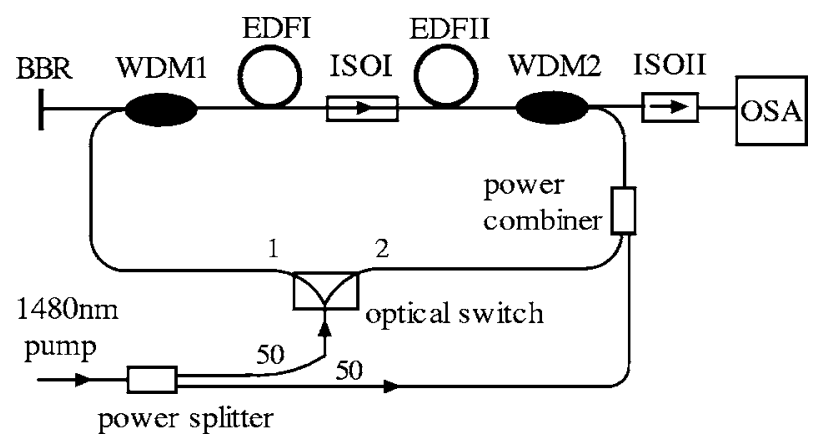

Fig. 4 Schematic diagram of the band selective ASE source using the compound pump technique. 


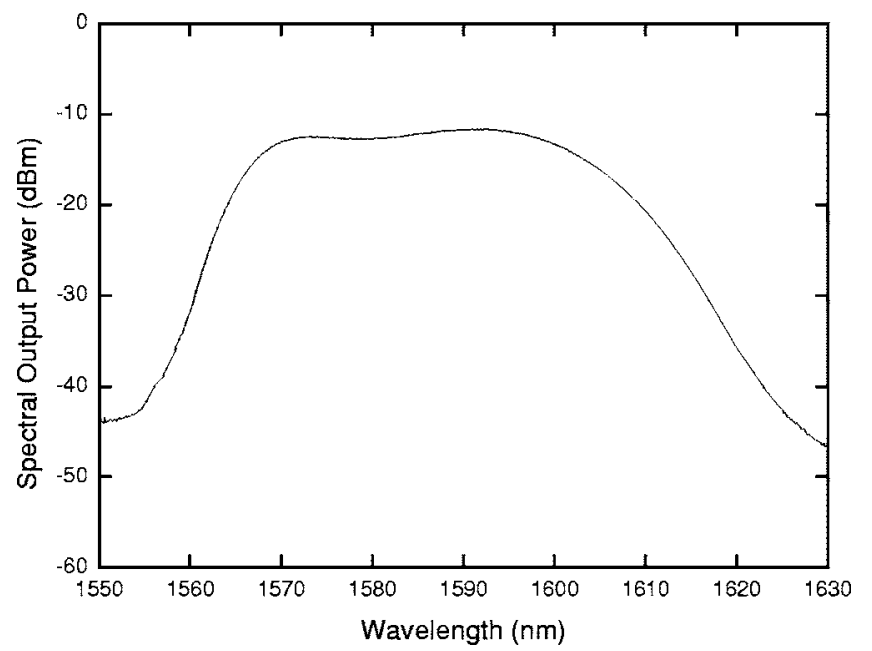

Fig. 5 Output L-band ASE spectrum when the optical switch is switching to port 1.

from C-band to the L-band, then increases the L-band gain in the forward direction, which provides a L-band spectrum at the output port. When the optical switch is switched to port 2, the forward pump is blocked. Namely, the EDFII is only pumped backward. The isolator (ISOI) in between the two fiber sections ensures that the C-band ASE will be obtained. ${ }^{5}$ Therefore, by switching the optical switch from port 1 to port 2, the operational band of the ASE design can be selected between C-band and L-band.

In this design, to obtain the L-band output when the optical switch switched to port 1, the EDF length should be selected properly because the output ASE operational band is mainly determined by the EDF length and the pump ratio of backward to forward pump power. According to our previous work on one-stage $\mathrm{C}+\mathrm{L}$-band ASE sources ${ }^{6}$ and the high-stability L-band erbium-doped SFS, ${ }^{10}$ when the EDF is bidirectionally pumped by two laser diodes, the longer wavelength ASE generated by the forward pump is substantially amplified by the ASE generated by the backward pump. Therefore, the OASIX3.0 software was first used for simulation. The Lucent LRL fiber with a peak absorption of $22-33 \mathrm{~dB} / \mathrm{m}$ at $1530 \mathrm{~nm}$ is used in both the simulations and experiments. The simulation results showed that the 19-m total fiber length is optimal for obtaining the flat L-band ASE with forward pump power of $50 \mathrm{~mW}$ and backward pump power of $50 \mathrm{~mW}$.

Figure 5 shows the experimental results of the output L-band ASE spectrum when the optical switch is switched to port 1 based on the above simulation parameters: $14-\mathrm{m}$ length of EDFI, 5-m length of EDFII. It can be seen from Fig. 5 that the ASE spectrum falls on L-band and is very flat. The output power of the L-band ASE is measured to be $24.5 \mathrm{~mW}$ and a $3-\mathrm{dB}$ bandwidth of about $40 \mathrm{~nm}$ is obtained without using any flattened filter. When the optical switch is switched to port 2, while keeping parameters of the design unchanged, the output spectrum falls on C-band with a power of $27.2 \mathrm{~mW}$, similar to Fig. 2. In fact, when the optical switch is switched to port 2, the ASE design becomes a backward-pumped configuration with 100-mW pump power. Namely, the output band is determined by the forward pump light. The C-band ASE is obtained without forward pump whereas L-band ASE is obtained with forward pump. It is important that the output power of the L-band ASE is effectively enhanced and is near to that of the C-band ASE with this configuration. It has been mentioned that the L-band ASE is obtained by the using the double-pass bidirectional pumped configuration, which is the best one for generating the L-band ASE. ${ }^{7}$ Therefore, the proposed selective C- and L-band ASE design not only provides users a selective C- or L-band ASE, but also provides a high output power with power balanced C- or L-band ASE source.

\section{Conclusion}

We have experimentally presented two novel methods to build up a selective dual-band ASE source that can operate in either the C- or the L-band region with the use of an optical switch. The selective ASE source can provide users the necessary band (C- or L-band) when needed without any form of upgrading or additional module attachment. The selective dual-band ASE source with the use of the compound pump technique provides users two selective bands with balanced and high output power, which means that it is more perfect and suitable for applications than using the backward ASE feedback technique. The selective nature of this ASE source design is desirable for the dualband DWDM device characterization and also has potential use as a selective upstream or downstream spectrum-sliced coarse WDM transceiver in the emerging metro networks.

\section{Acknowledgments}

This work was supported by Fujian Provincial Natural Science Foundation of China under Grant No. A0440009 and the Key Scientific and Technical Innovation Project of Xiamen University.

\section{References}

1. M. E. Bray, R. T. Elliot, and K. P. Jones, "Comparison of erbium amplifier measurement using a high power amplified spontaneous emission source or using an ITU grid," Opt. Fiber Commun. Conf. (OFC 2001), 3, WI2-1-WI2-3 (2001).

2. P. F. Wysocki, M. J. F. Digonnet, and B. Y. Kim, et al., "Characteristics of erbium-doped superfluorescent fiber sources for interferometric sensor applications," J. Lightwave Technol. 12, 550-567 (1994).

3. J. S. Lee, Y. C. Chung, and D. J. Digiovanni, "Spectrum-sliced fiber amplifier light source for multichannel WDM applications," IEEE Photonics Technol. Lett. 5, 1458-1461 (1993).

4. J. H. Lee, U.-C. Ryu, and N. Park, "Passive erbium-doped fiber seed photon generator for high power $\mathrm{Er}^{3+}$-doped fiber fluorescent sources with an $80 \mathrm{~nm}$ bandwidth," Opt. Lett. 24, 279-281 (1999).

5. R. P. Espindola, G. Ales, J. Park, and T. A. Strasser, "80 nm spectrally flattened high power erbium amplified spontaneous emission fiber source," Electron. Lett. 36(15), 1263-1265 (2000).

6. W. C. Huang, P. K. A. Wai, H. Y. Tam, et al., "One-stage erbium ASE source with $80 \mathrm{~nm}$ bandwidth and low ripples," Electron. Lett. 38(17), 956-957 (2002).

7. S.-C. Tsai, T.-C. Tsai, P.-C. Law, et al., "High pumping-efficiency L-band erbium-doped fiber ASE source using double-pass bidirectional-pumping configuration," IEEE Photonics Technol. Lett. 15(2), 197-199 (2003).

8. X. Wang and W. Huang, "High efficiency and stability L-Band erbium-doped superfluorescent fiber source," Proc. SPIE 5280, 221224 (2003)

9. W. Huang, H. Ming, and Z. Cai, et al., "A selective C and L-band amplified spontaneous emission source using a $1 \times 2$ optical switch," Chin. Phys. Lett. 22(5), 887-888 (2005).

10. X. Wang and W. Huang, "Wavelength stability optimization of L-band superfluorescent fiber source," Opt. Eng. 44, 060504 (2005). 